\title{
Mutable Checkpoints: A New Checkpointing Approach for Mobile Computing Systems
}

\author{
Guohong Cao, Member, IEEE, and Mukesh Singhal, Fellow, IEEE
}

\begin{abstract}
Mobile computing raises many new issues such as lack of stable storage, low bandwidth of wireless channel, high mobility, and limited battery life. These new issues make traditional checkpointing algorithms unsuitable. Coordinated checkpointing is an attractive approach for transparently adding fault tolerance to distributed applications since it avoids domino effects and minimizes the stable storage requirement. However, it suffers from high overhead associated with the checkpointing process in mobile computing systems. Two approaches have been used to reduce the overhead: First is to minimize the number of synchronization messages and the number of checkpoints; the other is to make the checkpointing process nonblocking. These two approaches were orthogonal previously until the Prakash-Singhal algorithm [28] combined them. However, we [8] found that this algorithm may result in an inconsistency in some situations and we proved that there does not exist a nonblocking algorithm which forces only a minimum number of processes to take their checkpoints. In this paper, we introduce the concept of "mutable checkpoint," which is neither a tentative checkpoint nor a permanent checkpoint, to design efficient checkpointing algorithms for mobile computing systems. Mutable checkpoints can be saved anywhere, e.g., the main memory or local disk of MHs. In this way, taking a mutable checkpoint avoids the overhead of transferring large amounts of data to the stable storage at MSSs over the wireless network. We present techniques to minimize the number of mutable checkpoints. Simulation results show that the overhead of taking mutable checkpoints is negligible. Based on mutable checkpoints, our nonblocking algorithm avoids the avalanche effect and forces only a minimum number of processes to take their checkpoints on the stable storage.
\end{abstract}

Index Terms-Mobile computing, coordinated checkpointing, causal dependency, nonblocking.

\section{INTRODUCTION}

distributed system is a collection of processes that $\mathrm{A}_{\text {communicate with each other by exchanging mes- }}$ sages. A mobile computing system is a distributed system where some processes are running on mobile hosts (MHs) that can move. To communicate with MHs, mobile support stations (MSSs) are added. An MSS communicates with other MSSs by wired networks, but it communicates with MHs by wireless networks. Due to the mobility of MHs and the constraints of wireless networks, there are some new issues [1], [20] that complicate the design of checkpointing algorithms:

- Changes in the location of an $M H$ complicate the routing of messages. Messages sent by an $\mathrm{MH}$ to another $M H$ may have to be rerouted since the destination $\mathrm{MH}$ may have moved. Although different routing protocols [2], [26] apply different techniques to address the mobility, generally speaking, locating an $\mathrm{MH}$ increases the communication delay and message complexity.

- G. Cao is with the Department of Computer Science and Engineering, Pennsylvania State University, University Park, PA 16802 E-mail: gcao@cse.psu.edu

- M. Singhal is with the Department of Computer and Information Science, Ohio State University, Columbus, OH 43210

E-mail:singhal@cis.ohio-state.edu

Manuscript received 1 Mar 2000; revised 1 Aug 2000; accepted 15 Aug 2000. For information on obtaining reprints of this article, please send e-mail to: tpds@computer.org, and reference IEEECS Log Number 112984.
- Due to the vulnerability of mobile computers to catastrophic failures, e.g., loss, theft, or physical damage, the disk storage on an $M H$ cannot be considered as the stable storage. A reasonable solution [1] is to utilize the stable storage at the MSSs to store checkpoints of the MHs. Thus, to take a checkpoint, an $M H$ has to transfer a large amount of data to its local MSS over the wireless network. Since the wireless network has low bandwidth and the $\mathrm{MHs}$ have relatively low computation power, the checkpointing algorithm should only force a minimum number of processes to take checkpoints.

- The battery at the $M H$ has limited life. To save energy, the $M H$ can power down individual components during periods of low activity [14]. This strategy is referred to as the doze mode operation. The $M H$ in doze mode is awakened on receiving a message. Therefore, energy conservation and low bandwidth constraints require the checkpointing algorithm to minimize the number of synchronization messages.

- MHs may disconnect from the network temporarily or permanently. The disconnection of MHs should not prevent the checkpointing process.

Coordinated checkpointing is a commonly used technique to prevent complete loss of computation upon a failure [7], [13], [19], [28], [34]. In this approach, the state of each process in the system is periodically saved on the stable storage, which is called a checkpoint of the process. To recover from a failure, the system restarts its execution from a previous consistent global checkpoint saved on the stable storage. In order to record a consistent global checkpoint, 
processes must synchronize their checkpointing activities. In other words, when a process takes a checkpoint, it asks (by sending checkpoint requests) all relevant processes to take checkpoints. Therefore, coordinated checkpointing suffers from high overhead associated with the checkpointing process.

Much of the previous work [12], [18], [19], [23] in coordinated checkpointing has focused on minimizing the number of synchronization messages and the number of checkpoints during checkpointing. However, these algorithms (called blocking algorithms) force all relevant processes in the system to block their computations during the checkpointing process. Checkpointing includes the time to trace the dependency tree and to save the states of processes on the stable storage, which may be long. Moreover, in mobile computing systems, due to the mobility of $\mathrm{MHs}$, a message may be routed several times before reaching its destination. Therefore, blocking algorithms may further degrade the performance of mobile computing systems [5], [13].

Recently, nonblocking algorithms [13], [30] have received considerable attention. In these algorithms, processes need not block during checkpointing by using a checkpointing sequence number to avoid inconsistencies. However, these algorithms [13], [30] require all processes in the computation to take checkpoints during the checkpointing, even though many of them may not be necessary. In mobile computing systems, since checkpoints need to be transfered to the stable storage at the MSSs over the wireless network, taking unnecessary checkpoints may waste a large amount of wireless bandwidth.

The Prakash-Singhal algorithm [28] was the first algorithm to combine these two approaches. More specifically, it only forces a minimum number of processes to take checkpoints and does not block the underlying computation during the checkpointing. However, we found that this algorithm may result in an inconsistency [7], [8] in some situations and we proved that there does not exist a nonblocking algorithm which forces only a minimum number of processes to take their checkpoints.

In this paper, we introduce the concept of "mutable checkpoint," which is neither a tentative checkpoint nor a permanent checkpoint, to design efficient checkpointing algorithms for mobile computing systems. Mutable checkpoints need not be saved on the stable storage and can be saved anywhere, e.g., the main memory or local disk of MHs. Thus, taking a mutable checkpoint avoids the overhead of transferring large amounts of data to the stable storage at $M S S$ s over the wireless network. We present techniques to minimize the number of mutable checkpoints. Simulation results show that the overhead of taking mutable checkpoints is negligible. Based on mutable checkpoints, our nonblocking algorithm forces only a minimum number of processes to take their checkpoints on the stable storage.

The rest of the paper is organized as follows: Section 2 develops the necessary background. In Section 3, we present a low-cost checkpointing algorithm for mobile computing systems. The correctness proof is provided in Section 4. In Section 5, we evaluate the performance of our algorithm. Related work is provided in Section 6. Section 7 concludes the paper.

\section{Preliminaries}

\subsection{Computation Model}

A mobile computing system consists of a large number of mobile hosts(MHs) [1] and relatively fewer static hosts called mobile support stations(MSSs). The MSSs are connected by a static wired network, which provides reliable FIFO delivery of messages. A cell is a logical or geographical area covered by an MSS. An $M H$ can directly communicate with an MSS by a reliable FIFO wireless channel only if it is present in the cell supported by the MSS.

The distributed computation we consider consists of $N$ processes denoted by $P_{0}, P_{1}, P_{2}, \cdots, P_{N}$ running concurrently on fail-stop $M H$ s or MSSs in the network. The processes do not share a common memory or a common clock. Message passing is the only way for processes to communicate with each other. The computation is asynchronous: Each process progresses at its own speed and messages are exchanged through reliable communication channels whose transmission delays are finite but arbitrary. The messages generated by the underlying distributed application will be referred to as computation messages. Messages generated by processes to advance checkpoints will be referred to as system messages.

Each checkpoint taken by a process is assigned a unique sequence number. The $i^{\text {th }}(i \geq 0)$ checkpoint of process $P_{p}$ is assigned a sequence number $i$ and is denoted by $C_{p, i}$. The $i^{\text {th }}$ checkpoint interval[25] of process $P_{p}$ denotes all the computation performed between its $i^{\text {th }}$ and $(i+1)^{\text {th }}$ checkpoint, including the $i^{\text {th }}$ checkpoint but not the $(i+1)^{\text {th }}$ checkpoint.

\subsection{Handling Mobility and Disconnections}

Due to the mobility of $M H s$, message transmission becomes complicated. Messages sent by an $\mathrm{MH}$ to another $\mathrm{MH}$ may have to be rerouted because the destination $M H$ may be disconnected from the old MSS and connected to a new MSS. Many routing protocols for the network layer have been proposed [2], [26], [33] to handle $M H$ mobility.

It should be noted that disconnection of an $M H$ is a voluntary operation [1], and frequent disconnections of $\mathrm{MHs}$ is an expected feature of the mobile computing environment. Unexpected disconnections due to battery failure, processor failure, or network failure are different from voluntary disconnection and are discussed in Section 3.6.

An $M H$ may get disconnected from the network for an arbitrary period of time. At the application level, the checkpointing algorithm may generate a request for the disconnected $\mathrm{MH}$ to take a checkpoint. Delaying a response to such a request until the $M H$ reconnects at some MSS may significantly increase the completion time of the checkpointing algorithm. So, we propose the following solution to deal with disconnections.

Note that only local events can take place at an $\mathrm{MH}$ during the disconnect interval. No message send or receive event occurs during this interval. Hence, no new dependencies with respect to other processes are created during this interval. The dependency relation of an $M H$ with the 
rest of the system, as reflected by its local checkpoint, is the same no matter when the local checkpoint is taken during the disconnect interval.

Suppose a mobile host $M H_{i}$ wants to disconnect from its local $M S S_{p} . M H_{i}$ takes a local checkpoint and transfers its local checkpoint to $M S S_{p}$ as disconnect_checkpoint ${ }_{i}$. If $M H_{i}$ is asked to take a checkpoint during the disconnect interval, $M S S_{p}$ converts disconnect_checkpoint $t_{i}$ into $M H_{i}$ 's new checkpoint and uses the message dependency information of $M H_{i}$ to propagate the checkpoint request. $M H_{i}$ also sends a disconnect $(s n)$ message to $M S S_{p}$ on the $M H$-to-MSS channel supplying the sequence number $s n$ of the last message received on the $M S S$-to- $M H$ channel. On receipt of $M H_{i}$ 's disconnect(sn), $M S S_{p}$ knows the last message that $M H_{i}$ received from it and buffers all computation messages received until the end of the disconnect interval.

Later, suppose $M H_{i}$ reconnects at an $M S S$, say $M S S_{q}$. If $M H_{i}$ knows the identity of its last $M S S$, say $M S S_{p}$, it sends a reconnect $\left(M H_{i}, M S S_{q}\right)$ message to $M S S_{p}$ through $M S S_{q}$. If $M H_{i}$ lost the identity of its last MSS for some reason, $M H_{i}$ 's reconnect request is broadcast over the network. On receiving the reconnect request, $M S S_{p}$ transfers all the support information (the checkpoint, dependency vector, buffered messages, etc.) of $M H_{i}$ to $M S S_{q}$ and removes all the information related to the disconnection. Then, $M S S_{q}$ forwards all the support information to $M H_{i}$. When the data sent by $M S S_{p}$ arrives at $M H_{i}, M H_{i}$ processes the buffered messages. If $M S S_{p}$ has taken a checkpoint for $M H_{i}, M H_{i}$ clears its message dependency information before processing the buffered messages. After these activities, the reconnect routine terminates and the relocated mobile host $M H_{i}$ resumes normal communication with other MHs (or MSSs) in the system.

\subsection{The Basic Idea behind Nonblocking Algorithms}

Most existing coordinated checkpointing algorithms [12], [19], [23] rely on the two-phase commit protocol [15] and save two kinds of checkpoints on the stable storage: tentative and permanent. In the first phase, the initiator takes a tentative checkpoint and forces all relevant processes to take tentative checkpoints. Each process informs the initiator whether it succeeded in taking a tentative checkpoint. A process may refuse to take a checkpoint depending on its underlying computation. After the initiator has received positive replies from all relevant processes, the algorithm enters the second phase. If the initiator learns that all processes have successfully taken tentative checkpoints, it asks them to make their tentative checkpoints permanent; otherwise, it asks them to discard them. A process, on receiving the message from the initiator, acts accordingly. Note that, after a process takes a tentative checkpoint in the first phase, it remains blocked until it receives the decision from the initiator in the second phase.

A nonblocking checkpointing algorithm does not require any process to suspend its underlying computation. When processes do not suspend their computations, it is possible for a process to receive a computation message from another process which is already running in a new checkpoint interval. If this situation is not properly handled, it may result in an inconsistency. For example, in Fig. $1, P_{2}$

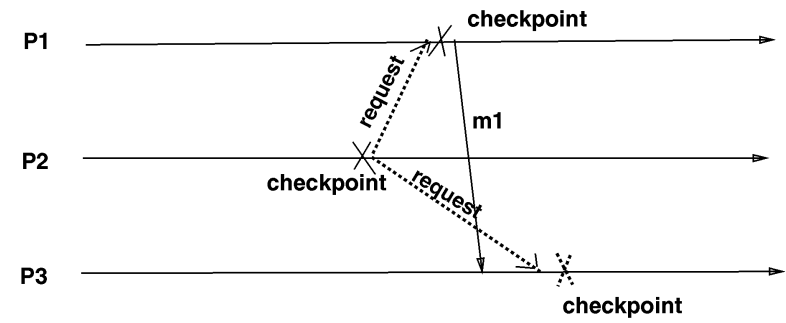

Fig. 1. Inconsistent checkpoints

initiates a checkpointing process. After sending checkpoint requests to $P_{1}$ and $P_{3}, P_{2}$ continues its computation. $P_{1}$ receives the checkpoint request and takes a new checkpoint, then it sends $m 1$ to $P_{3}$. Suppose $P_{3}$ receives the checkpoint request from $P_{2}$ after receiving $m 1$. The recorded checkpoints are not consistent with each other since $m 1$ is an orphan message, i.e., a message whose receive event is recorded in the state of the destination process, but its send event is lost [19], [32].

Most nonblocking algorithms [13], [24], [30] use a Checkpoint Sequence Number (csn) to avoid inconsistencies. More specifically, a process is forced to take a checkpoint if it receives a computation message whose csn is greater than its local csn. In Fig. 1, $P_{1}$ increases its $c s n$ after it takes a checkpoint and appends the new csn to $m 1$. When $P_{3}$ receives $m 1$, it takes a checkpoint before processing $m 1$ because the csn appended to $m 1$ is larger than its local csn.

This scheme works only when every process in the computation can receive each checkpoint request and increases its own csn. Since the Prakash-Singhal algorithm [28] only forces a part of the processes to take checkpoints, the $c s n$ of some processes may be out-of-date, and may not be able to avoid inconsistencies. The Prakash-Singhal algorithm attempts to solve this problem by having each process maintain an array to save the $c s n$, where $c s n_{i}[j]$ represents the $\operatorname{csn}$ of $P_{j}$ that $P_{i}$ expects. Note that $P_{i}^{\prime}$ s $\operatorname{csn} n_{i}[i]$ may be different from $P_{j}^{\prime}$ s $c s n_{j}[i]$ if there has been no communication between $P_{i}$ and $P_{j}$ for several checkpoint intervals. By using $c s n$ and the initiator identification number, they claim that their nonblocking algorithm can avoid inconsistencies and minimize the number of checkpoints during checkpointing. However, we showed that this algorithm may result in an inconsistency [7], [8], and we have proven that there does not exist a nonblocking algorithm which forces only a minimum number of processes to take their checkpoints [7], [8]. Since the proof is not the major concern of this paper, we only briefly mention the basic idea using an example.

\subsection{Impossibility of Checkpointing}

In Fig. 2, assume messages $m 6$ and $m 7$ do not exist. To initiate a checkpointing process, $P_{1}$ takes checkpoint $C_{1,1}$ and sends checkpoint requests to $P_{3}$ and $P_{4}$ (not illustrated in the figure) since it depends on them. When $P_{4}$ receives the checkpoint request, it takes a checkpoint and sends a checkpoint request to $P_{5}$. For the same reason, $P_{5}$ takes a checkpoint and sends a checkpoint request to $P_{2}$. $P_{2}$ must take this checkpoint before processing $m 5$; otherwise, $m 5$ will become an orphan. Things are complicated if we 


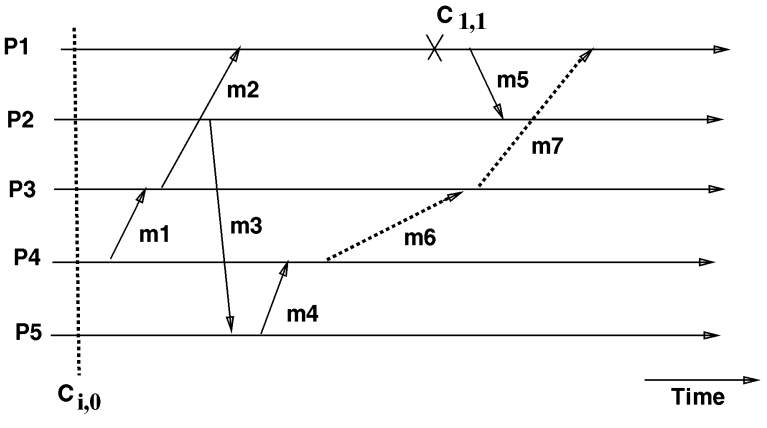

Fig. 2. Tracing the dependency

consider another situation. Suppose $m 4$ does not exist. In this case, $P_{2}$ will not receive a checkpoint request associated with checkpoint $C_{1,1}$, and it should not take a checkpoint before processing $m 5$ in order to minimize the number of checkpoints. Therefore, when $P_{2}$ receives $m 5$, it has to decide whether to take a checkpoint before processing $m 5$. In other words, $P_{2}$ has to know if it will receive a checkpoint request associated with $C_{1,1}$ in the future when it receives $m 5$. However, if the checkpointing process is nonblocking, there is not enough information for $P_{2}$ to look into the future.

The problem arises due to the dependency created by the message $m 4$. Because of $m 4$, there is a new dependency between $P_{1}$ and $P_{2}$ such that $P_{2}$ will receive a checkpoint request associated with $C_{1,1}$. There are two possible approaches for $P_{2}$ to get the information about this new dependency (called the z-dependency [7], [8]).

Approach 1 (Tracing the in-coming messages): In this approach, $P_{2}$ obtains the new z-dependency information from $P_{1}$. Then, $P_{1}$ has to know the $\mathrm{z}$-dependency information before it sends $m 5$ and appends the z-dependency information to $m 5$. In Fig. 2, $P_{1}$ cannot get the new z-dependency information unless $P_{4}$ notifies $P_{1}$ of the new Z-dependency information when $P_{4}$ receives $m 4$. There are two ways for $P_{4}$ to notify $P_{1}$ of the new z-dependency information: First is to broadcast the z-dependency information (not illustrated in the figure); the other is to send the z-dependency information on an extra message $m 6$ to $P_{3}$, which in turn sends it to $P_{1}$ on $m 7$. Both of them dramatically increase the message overhead. Since the algorithm does not block the underlying computation, it is possible that $P_{1}$ receives $m 7$ after it sends out $m 5$ (as shown in the figure) and, hence, $P_{2}$ is still not guaranteed to get the $\mathrm{z}$-dependency information when it receives $m 5$.

Approach 2 (Tracing the out-going messages): In this approach, since $P_{2}$ sends message $m 3$ to $P_{5}, P_{2}$ hopes to obtain the new z-dependency information from $P_{5}$. $P_{5}$ has to know the new $\mathrm{z}$-dependency information and it must send an extra message (not shown in the figure) to notify $P_{2}$. Similarly, $P_{5}$ needs to get the new z-dependency information from $P_{4}$ which comes from $P_{3}$ and, finally, from $P_{1}$. This requires many more extra messages than Approach 1. Similar to Approach 1, $P_{2}$ is still not guaranteed to get the $\mathrm{z}$-dependency information in time since the computation is in progress.

In conclusion, there does not exist a nonblocking algorithm that forces only a minimum number of processes to take their checkpoints.

\section{A Checkpointing Algorithm Based on Mutable CheckPoints}

In this section, we present our checkpointing algorithm, which neither blocks the underlying computation nor forces all processes to take checkpoints.

\subsection{The Basic Idea}

\subsubsection{The Basic Schemes}

A simple nonblocking scheme for checkpointing is as follows: When a process $P_{i}$ sends a message, it piggybacks the current value of $c s n_{i}[i]$ (csn was explained in Section 2.3). When a process $P_{j}$ receives a message $m$ from $P_{i}, P_{j}$ processes the message if $m . c s n \leq c s n_{j}[i]$; otherwise, $P_{j}$ takes a checkpoint, updates its $\operatorname{csn}\left(c s n_{j}[i]=\right.$ m.csn $)$, and processes the message. This method may result in a large number of checkpoints. Moreover, it may lead to an avalanche effect in which processes in the system recursively ask others to take checkpoints.

For example, in Fig. 3, to initiate a checkpointing process, $P_{2}$ takes its own checkpoint and sends checkpoint requests to $P_{1}, P_{3}$ and $P_{4}$. When $P_{2}$ 's request reaches $P_{4}, P_{4}$ takes a checkpoint and sends message $m 3$ to $P_{3}$. When $m 3$ arrives
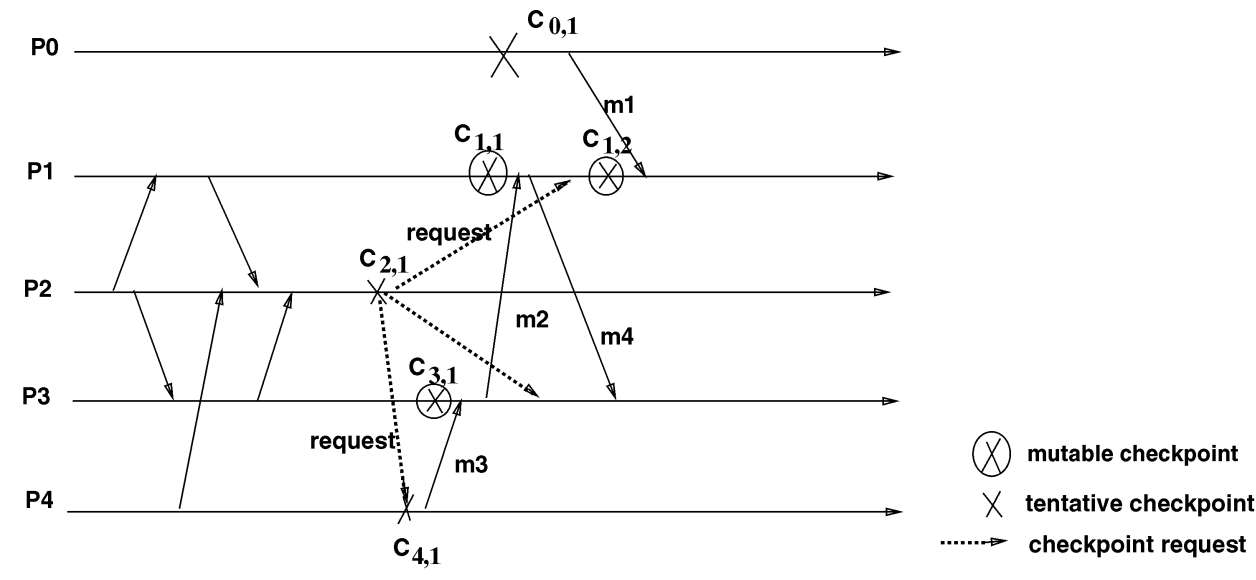

....... checkpoint request

Fig. 3. An example of checkpointing 
at $P_{3}, P_{3}$ takes a checkpoint before processing it since $m 3 . c s n>\operatorname{csn}_{3}[4]$. For the same reason, $P_{1}$ takes a checkpoint before processing $m 2$.

$P_{0}$ has not communicated with other processes before it takes a local checkpoint. Later, it sends message $m 1$ to $P_{1}$. $P_{1}$ takes the checkpoint $C_{1,2}$ before processing $m 1$ since $P_{0}$ has taken a checkpoint which has a checkpoint sequence number larger than $P_{1}$ expected. Then, $P_{1}$ requires $P_{3}$ to take another checkpoint (not shown in the figure) due to $m 2$ and $P_{3}$ in turn asks $P_{4}$ to take another checkpoint (not shown in the figure) due to $m 3$. If $P_{4}$ had received messages from other processes after it sent $m 3$, those processes would have been forced to take checkpoints. This chain may never end.

We reduce the number of checkpoints based on the following observation: In Fig. 3, if $m 4$ does not exist, it is not necessary for $P_{1}$ to take $C_{1,2}$ since checkpoint $C_{1,1}$ is consistent with the rest of checkpoints. Based on this observation, we get the following revised scheme.

When a process $P_{j}$ receives a message $m$ from $P_{i}, P_{j}$ only takes a checkpoint when m.csn $>c s n_{j}[i]$ and $P_{j}$ has sent at least one message in the current checkpoint interval.

In Fig. 3, if $m 4$ does not exist, $C_{1,2}$ is not necessary according to the revised scheme. However, if $m 4$ exists, the revised scheme still results in a large number of checkpoints and may result in an avalanche effect.

\subsubsection{The Enhanced Scheme}

We now present the basic idea of our scheme that eliminates avalanche effects during checkpointing. From Fig. 3, we make two observations:

Observation 1. It is not necessary to take checkpoint $C_{1,2}$ even though $m 4$ exists since $P_{1}$ will not receive a checkpoint request associated with $C_{0,1}$. Note that $m 4$ will not become an orphan even though it does not take checkpoint $C_{1,2}$.

Observation 2. From Section 2.4, $P_{1}$ does not have enough information to know if it will receive a checkpoint request associated with $C_{0,1}$ when $P_{1}$ receives $m 1$.

These observations imply that $C_{1,2}$ is unnecessary but still unavoidable. Thus, there are two kinds of checkpoints in response to computation messages. In Fig. 3, $C_{1,1}$ is different from $C_{1,2}$. $C_{1,1}$ is a checkpoint associated with the initiator $P_{2}$ and $P_{1}$ will receive a checkpoint request for the checkpointing initiated by $P_{2} . C_{1,2}$ is a checkpoint associated with the initiator $P_{0}$, but $P_{1}$ will not receive a checkpoint request for the checkpointing initiated by $P_{0}$ in the future. To avoid inconsistency, $P_{1}$ should keep $C_{1,1}$ when it receives $P_{2}$ 's request. However, $P_{1}$ can discard $C_{1,2}$ after the checkpointing initiated by $P_{0}$ terminates $\left(C_{0,1}\right.$ becomes a permanent checkpoint) since, at that time, $P_{1}$ is sure that it will not receive any checkpoint request associated with $P_{0}$ 's initiation. Moreover, if $P_{0}$ has finished its checkpointing process before it sends $m 1, P_{1}$ does not need to take checkpoint $C_{1,2}$.

We introduce a new concept, called mutable checkpoint, to reflect the essence of the checkpoints (like $C_{1,1}, C_{1,2}$ ) triggered by computation messages. A mutable checkpoint is neither a tentative checkpoint nor a permanent check-

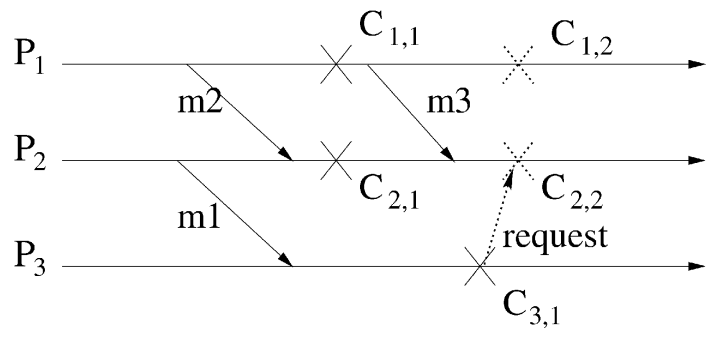

Fig. 4. Further reduce the number of checkpoints

point, but it can be turned into a tentative checkpoint. When a process takes a mutable checkpoint, it does not send checkpoint requests to other processes and it does not need to save the checkpoint on the stable storage. It can save the mutable checkpoint anywhere, e.g., in the main memory or the local disk of $M H$ s. Suppose a process $P_{i}$ has taken a mutable checkpoint. When $P_{i}$ receives a checkpoint request, it transfers the mutable checkpoint to the stable storage and forces all dependent processes to take tentative checkpoints. In this way, $P_{i}$ turns its mutable checkpoint into a tentative checkpoint. If $P_{i}$ does not receive the checkpoint request after the checkpointing activity terminates (implementation details will be discussed in the next section), it discards the mutable checkpoint.

In Fig. 3, when $m 2$ arrives at $P_{1}, P_{1}$ takes a mutable checkpoint $C_{1,1}$ before processing it since $m 2$.csn $>c s n_{1}[3]$. $C_{1,1}$ is turned into a tentative checkpoint when $P_{1}$ receives the checkpoint request sent by $P_{2}$. If $P_{0}$ has finished its checkpointing activity before it sends $m 1, P_{1}$ does not need to take a mutable checkpoint $C_{1,2}$. Otherwise, $P_{1}$ takes a mutable checkpoint $C_{1,2}$, which will be discarded when $P_{0}$ 's checkpointing terminates. Since $C_{1,2}$ is a mutable checkpoint, it does not force $P_{3}$ to take a new checkpoint. Thus, our scheme avoids the avalanche effect and significantly reduces the checkpointing overhead. If there is no ambiguity, we simply refer to a tentative or permanent checkpoint as a checkpoint.

\subsubsection{Further Reduction in the Number of Checkpoints}

In the above scheme, a process may receive unnecessary checkpoint requests and may take unnecessary checkpoints. As shown in Fig. 4, $P_{2}$ initiates a checkpointing process by taking a checkpoint $C_{2,1}$ and forces $P_{1}$ to take a checkpoint $C_{1,1}$ (due to $m 2$ ). Later, to initiate a checkpointing process, $P_{3}$ takes a checkpoint $C_{3,1}$ and sends a request to $P_{2}$ due to $m 1$. When $P_{2}$ receives the request, it takes a checkpoint $C_{2,2}$ and forces $P_{1}$ to take a checkpoint $C_{1,2}$. However, $C_{2,2}$ and $C_{1,2}$ are not necessary since $m 1$ is not an orphan even though $C_{1,2}$ and $C_{2,2}$ do not exist.

These unnecessary checkpoints can be avoided by the following method: When a process $P_{i}$ sends a checkpoint request to $P_{j}$, it attaches $\operatorname{csn} n_{i}[j]$ to the request. On receiving the request, $P_{j}$ compares the attached $c s n_{i}[j]$ (req_csn) with its own $c s n_{j}[j]$. If $c s n_{j}[j]>$ req_csn (i.e., $P_{j}$ has recorded the sending of the message which creates the dependency between $P_{i}$ and $P_{j}$ ), $P_{j}$ does not need to take a checkpoint; otherwise, it takes a checkpoint. In Fig. 4 , when $P_{3}$ sends a request to $P_{2}$, it attaches $c s n_{3}[2]=0$ to the request. When $P_{2}$ receives the request, $c s n_{2}[2]$ has been increased to 1 due to 
$C_{2,1}$. Thus, $P_{2}$ ignores this request and does not take checkpoint $C_{2,2}$ and, subsequently, $P_{2}$ does not force $P_{1}$ to take checkpoint $C_{1,2}$.

\subsection{Notations and Data Structures}

The following notations and data structures are used in our algorithm:

- $\quad R_{i}$ : An array of $n$ bits at process $P_{i} . R_{i}[j]=1$ represents that $P_{i}$ receives a computation message from $P_{j}$ in the current checkpoint interval.

- $\quad c s n_{i}$ : An array of $n$ checkpoint sequence numbers (csn) at each process $P_{i}$. $\quad c s n_{i}[j]$ represents the checkpoint sequence number of $P_{j}$ that $P_{i}$ knows. In other words, $P_{i}$ expects to receive a message from $P_{j}$ with the checkpoint sequence number $c s n_{i}[j]$.

- weight: A nonnegative variable of type real with a maximum value of 1 . It is used to detect the termination of the checkpointing algorithm as in [16].

- trigger $_{i}$ : A tuple (pid, inum) maintained by each process $P_{i}$. pid indicates the checkpointing initiator that triggered the latest checkpointing process. inum indicates the csn at process pid when it took its own local checkpoint on initiating the checkpointing.

- $\operatorname{sent}_{i}$ : A Boolean which is set to 1 if $P_{i}$ has sent a message in the current checkpoint interval.

- $\quad$ cp_state $_{i}$ : A Boolean which is set to 1 if $P_{i}$ is in the checkpointing process.

- old_csn: A variable used to save the csn of the current tentative (permanent) checkpoint.

- $\quad C P_{i}$ : A record maintained by each process $P_{i}$. Each record has the following fields:

mutable: the mutable checkpoint of $P_{i}$.

$R$ : $P_{i}$ 's own Boolean vector before it takes the current mutable checkpoint.

trigger: the trigger which is associated with the current mutable checkpoint.

sent: $P_{i}$ 's own sent before it takes the current mutable checkpoint.

csn is initialized to an array of 0 s at all processes. The trigger tuple at process $P_{i}$ is initialized to $(i, 0)$. The weight and cp_state at a process is initialized to 0 . When a process $P_{i}$ sends a computation message, it appends its $\operatorname{csn}_{i}[i]$ to the message. Also, $P_{i}$ checks if $c p_{-}$state $_{i}$ is equal to 1 . If so, it appends its trigger to the computation message.

When a process $P_{j}$ receives a checkpoint request from $P_{i}$, we say " $P_{j}$ inherits a request from $P_{i}$ " if and only if old_csn ${ }_{j} \leq r e q_{-} c s n$ (req_csn is appended with the request) and $P_{j}$ takes a tentative checkpoint. In this definition, we use old_csn $n_{j}$ instead of $c s n_{j}[j]$ used in Section 3.1 since $\operatorname{csn}_{j}[j]$ is also increased when taking a mutable checkpoint, but we need to compare req_csn with the $c s n$ of the current tentative (permanent) checkpoint.

\subsection{The Checkpointing Algorithm}

In this section, we present our nonblocking checkpointing algorithm. To clearly present the algorithm, we assume that, at any time, at most one checkpointing is in progress. In
Section 3.5, we extend the algorithm for concurrent invocations.

\subsubsection{Checkpointing Initiation}

Any process can initiate a checkpointing process. When a process $P_{i}$ initiates a checkpointing process, it takes a local checkpoint, increments its $\operatorname{csn}_{i}[i]$, sets weight $t_{i}$ to 1 , sets cp_state $_{i}$ to 1 , and stores its own identifier and the new $\operatorname{csn}_{i}[i]$ in its trigger. Then, it sends a checkpoint request to each process $P_{j}$ such that $R_{i}[j]=1$ and resumes its computation. Each request carries the trigger of the initiator, $R_{i}$ and a portion of the weight of the initiator, whose weight is decreased by an equal amount.

\subsubsection{Reception of a Checkpoint Request}

When a process $P_{i}$ receives a request from $P_{j}$, it first compares req_csn with its old_csn to see if it needs to inherit the request. If $P_{i}$ does not need to inherit the request, it sends the appended weight to the initiator and then exits. Otherwise, it updates its $c s n$ and cp_state and compares $P_{j}$.trigger (msg_trigger) with $P_{i}$.trigger (own_trigger). If msg_trigger = own_trigger (implying that $P_{i}$ has already taken a checkpoint for this checkpointing), $P_{i}$ checks if there is a mutable checkpoint which has a trigger identical to msg_trigger. If not, $P_{i}$ sends the appended weight to the initiator; otherwise, $P_{i}$ saves the mutable checkpoint on the stable storage (the mutable checkpoint is turned into a tentative checkpoint) and then propagates the request. If $P_{i}$ propagates the request to all processes on which it depends, it may result in a large number of redundant system messages since some processes on which $P_{i}$ depends may have received the request from other processes. The Koo-Toueg algorithm [19] uses this approach and its system message overhead can be as large as $O\left(N^{2}\right)$, where $N$ is the number of processes in the system. On the other hand, only propagating the request to processes on which $P_{i}$ depends, but $P_{j}$ (the sender) does not, may not work since receiving a request does not necessarily mean that the process inherits the request. We solve this problem by attaching some information (csn and $R$ which are saved in a structure called $M R$ in the algorithm) to the request. $P_{i}$ only propagates the request to each process $P_{k}$ on which $P_{i}$ depends, but $P_{k}$ may not have inherited the request; that is, if $P_{i}$ knows (by $M R$ ) some other process has sent the request to $P_{k}$ with $r e q_{-} c s n \geq c s n_{i}[k]$ (req-csn is appended with the request and saved in $M R[k] . c s n)$, it does not need to send the request to $P_{k}$; otherwise, it has to send the request since $P_{k}$ may inherit the request from $P_{i}$. Also, $P_{i}$ appends the initiator's trigger and a portion of the received weight to all those requests. Then, $P_{i}$ sends a reply to the initiator with the weight equal to the remaining weight and resumes its underlying computation. If msg_trigger $\neq$ own_trigger, $P_{i}$ takes a tentative checkpoint, increases its $c s n_{i}[i]$, and propagates the request as above. At last, $P_{i}$ clears $R_{i}$ and sent $t_{i}$, sends a reply to the initiator with the remaining weight, and then resumes its underlying computation. 


\subsubsection{Computation Messages Received during Checkpointing}

When $P_{i}$ receives a computation message from $P_{j}, P_{i}$ compares $m . c s n$ with its local $\operatorname{csn} n_{i}[j]$. If $m . c s n \leq c s n_{i}[j]$, the message is processed and no checkpoint is taken. Otherwise, it implies that $P_{j}$ has taken a checkpoint before sending $m . P_{i}$ updates its $c s n_{i}[j]$ to $m . c s n$ and checks if the following conditions are satisfied:

- Condition 1: $P_{j}$ is in the checkpointing process before sending $m$.

- Condition 2: $P_{i}$ has sent a message since last checkpoint.

- Condition 3: $P_{i}$ has not taken a checkpoint associated with the initiator (in the msg_trigger).

If all of them are satisfied, $P_{i}$ takes a mutable checkpoint and updates its data structures such as $c s n, C P, R$, cp_state, and sent. If only Condition 1 is satisfied, $P_{i}$ only increases $\operatorname{csn}_{i}[i]$ and sets $c p \_s t a t e_{i}$ to 1 .

\subsubsection{Termination and Garbage Collection}

The initiator adds weights received in all reply messages to its own weight. When its weight becomes equal to 1 , it concludes that all processes involved in the checkpointing have taken their tentative checkpoints and, hence, it broadcasts commit messages to all processes in the system. If a process has taken a tentative checkpoint, on receiving the commit message, it makes its tentative checkpoint permanent and clears $c p_{-} s t a t e$. Other processes also clear their cp_state and discard mutable checkpoints if there is any. Note that, when a process discards its mutable checkpoints, it updates its $R$ and sent.

\subsubsection{Instead of Broadcasting commit Messages to All Processes}

In [6], the initiator only sends commit messages to those processes from which it has received reply messages. However, to clear $c p \_s t a t e$, each process needs to maintain a history of the processes to which it has sent messages when its $c p \_s t a t e$ is equal to 1 . Also, it notifies them to clear their cp_state. There is a trade-off between these two approaches. If there are many communications among processes during the last checkpoint interval, the broadcast approach is better. On the other hand, if there are only a limited number of message exchanges during the last checkpoint interval, the update approach [6] is better. To obtain the advantages of both approaches, the initiator can use a counter to save the number of processes that have taken checkpoints. If the counter is larger than a value (a system tuning parameter), the broadcast approach is used; otherwise, the update approach is used. Since this paper concentrates on reducing the overhead of saving checkpoints, we simply use the broadcast approach.

Actions taken when $P_{i}$ sends a computation message to $P_{j}$ : if $c p \_s t a t e_{i}=1$

then $\operatorname{send}\left(P_{i}\right.$, message, $\operatorname{csn}_{i}[i]$, own_trigger $)$; sent $t_{i}:=1$;

else send $\left(P_{i}\right.$, message, $\left.\operatorname{csn}_{i}[i], N U L L\right)$; $\operatorname{sent}_{i}:=1$;

Actions for the initiator $P_{j}$ :

increment $\left(\operatorname{csn}_{j}[j]\right)$; own_trigger $:=\left(P_{j}, \operatorname{csn}_{j}[j]\right)$;

cp_state $_{j}:=1$; weight w $_{j}:=0$; for $\mathrm{k}:=0$ to $N$ do $M R[k] . c s n:=0 ; M R[k] . R:=0$;

$M R[j] . \operatorname{csn}:=\operatorname{csn}_{j}[j] ; M R[j] . R:=1$;

$\operatorname{prop} \_c p\left(R_{j}, M R, P_{j}\right.$, own_trigger, 1.0);

take a local checkpoint (on the stable storage);

$$
\text { old_csn }_{j}:=\operatorname{csn}_{j}[j] \text {; } \operatorname{sent}_{j}:=0 \text {; reset } R_{j} \text {; }
$$

Actions at process, $P_{i}$, on receiving a checkpoint request from $P_{j}$ :

receive $\left(P_{j}\right.$, request, $M R$,recv_csn, msg_trigger, req_csn,recv_weight);

$\operatorname{csn}_{i}[j]:=$ recv_csn;

if old_csn $_{i}>$ req_csn

then send $\left(P_{i}\right.$, reply, recv_weight $)$ to the initiator; return;

cp_state $_{i}:=1$;

if msg_trigger $=$ own_trigger

then if $C P_{i}$.trigger $=$ msg_trigger

then prop_cp $\left(C P_{i} . R, M R, P_{i}\right.$, msg_trigger, recv_weight); save $C P_{i}$.mutable on the stable storage; old_csn $_{i}:=\operatorname{csn}_{i}[i] ; C P_{i}:=N U L L$; send $\left(P_{i}\right.$, reply, weight $\left.t_{i}\right)$ to the initiator;

else send $\left(P_{i}\right.$, reply, recv_weight $)$ to the initiator;

else increment $\left(\operatorname{csn}_{i}[i]\right)$; own_trigger $:=$ msg_trigger;

prop_cp $\left(R_{i}, M R, P_{i}\right.$, msg_trigger, recv_weight);

take a local checkpoint (on the stable storage);

old_csn $_{i}:=\operatorname{csn}_{i}[i]$;

$\operatorname{send}\left(P_{i}\right.$, reply, weight $\left.t_{i}\right)$ to the initiator;

sent $_{i}:=0$; reset $R_{i}$;

Actions at process $P_{i}$, on receiving a computation message from $P_{j}$ :

receive $\left(P_{j}, m\right.$,recv_csn, msg_trigger $)$;

if recv_csn $\leq c s n_{i}[j]$

then $R_{i}[j]:=1$; process the message;

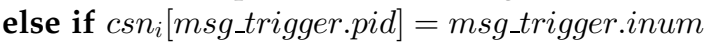

then $\operatorname{csn}_{i}[j]:=$ recv_csn $R_{i}[j]:=1$; process the message;

else $c s n_{i}[j]:=r e c v_{-} c s n$;

if msg_trigger $\neq N U L L \wedge$ sent $_{i}=1 \wedge$ msg_trigger $\neq$ own_trigger

then take a local checkpoint, save it in $C P_{i}$.mutable;

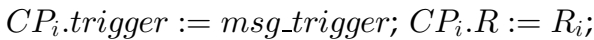
$C P_{i}$.sent $:=$ sent $_{i} ;$ sent $_{i}:=0$; reset $R_{i}$;

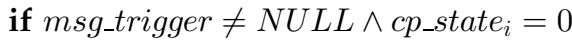

then $c_{p} \_s t a t e_{i}:=1$; increment $\left(\operatorname{csn}_{i}[i]\right)$; own_trigger :=msg_tigger;

$R_{i}[j]:=1$; process the message;

prop_cp $\left(R_{i}, M R, P_{i}\right.$, msg_trigger, recv_weight $)$

weight $_{i}:=$ recv_weight $_{i}$;

for $\mathrm{k}:=0$ to $N$ do temp[k].csn $:=\max \left(M R[k] . c s n, c s n_{i}[k]\right)$; temp $[\mathrm{k}] . \mathrm{R}:=\max \left(M R[k] \cdot R, R_{i}[k]\right)$;

for any $P_{k}$, such that $\left(R_{i}[k]=1\right) \wedge$ $\left(\max \left(M R[k] . c s n, \operatorname{csn}_{i}[k]\right) \neq M R[k] . c s n\right)$

weight $_{i}:=$ weight $_{i} / 2$;

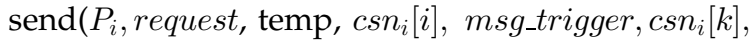
weight $\left._{i}\right)$;

Actions in the second phase for the initiator $P_{i}$ : receive $\left(P_{j}\right.$, reply, recv_weight $)$; 
weight $_{i}:=$ weight $_{i}+$ recv_weight;

if weight $_{i}=1$

then cp_state $_{i}:=0$; broadcast(commit,msg_trigger);

Actions at other process $P_{j}$ on receiving a broadcast message:

receive(commit, msg_trigger);

$c s n_{j}[$ msg_trigger.pid $]=$ msg_trigger.inum;

$c p_{-}$state $_{j}:=0$;

if $C P_{j}$.trigger $=$ msg_trigger $\wedge C P_{j} \neq N U L L$

then sent $_{j}:=$ sent $_{j} \cup C P_{j}$.sent; $R_{j}:=R_{j} \cup C P_{j} . R$;

$C P_{j}:=N U L L$;

if there is a tentative checkpoint associated with msg_trigger, $^{-}$ make it permanent;

\subsection{An Example}

The basic idea of the algorithm can be better understood by the example shown in Fig. 3. To initiate a checkpointing process, $P_{2}$ takes its own checkpoint and sends checkpoint requests to $P_{1}, P_{3}$, and $P_{4}$ since $R_{2}[1]=1, R_{2}[3]=1$, and $R_{2}[4]=1$. When $P_{2}$ 's request reaches $P_{4}, P_{4}$ takes a checkpoint and sends message $m 3$ to $P_{3}$. When $m 3$ arrives at $P_{3}, P_{3}$ takes a mutable checkpoint before processing the message since $m 3 . c s n>c s n_{3}[4]$ and $P_{3}$ has sent a message during the current checkpoint interval. For the same reason, $P_{1}$ takes a mutable checkpoint before processing $m 2$.

$P_{0}$ did not communicate with another process before it took the local checkpoint. Later, it sends a message $m 1$ to $P_{1}$. If $P_{0}$ has finished its checkpointing process before it sends $m 1, P_{1}$ does not need to take the checkpoint $C_{1,2}$. Otherwise, $P_{1}$ takes a mutable checkpoint $C_{1,2}$ before processing $m 1$.

When $P_{1}$ receives the checkpoint request from $P_{2}$, since $C_{1,1}$ is a mutable checkpoint associated with $P_{2}, P_{1}$ turns $C_{1,1}$ into a tentative checkpoint by saving it on the stable storage. Similarly, $P_{3}$ converts $C_{3,1}$ to a tentative checkpoint when it receives the checkpoint request from $P_{2}$. Finally, the checkpointing initiated by $P_{2}$ terminates when the checkpoints $C_{1,1}, C_{2,1}, C_{3,1}$, and $C_{4,1}$ are made permanent. $P_{1}$ discards $C_{1,2}$ when it makes checkpoint $C_{1,1}$ permanent or receives $P_{0}$ 's commit, whichever is earlier.

\subsection{Multiple Concurrent Initiations}

The simplest way to handle concurrent checkpoint initiations is to use the techniques in [19]. When a process $P_{i}$ receives a checkpoint request from $P_{j}$ while executing the checkpoint algorithm, $P_{i}$ ignores $P_{j}$ 's checkpoint request or defers the request until it finishes its current checkpointing. If $P_{i}{ }^{\prime}$ s checkpoint request is ignored by a process, $P_{i}$ has to abort its checkpointing efforts, which results in poor performance. A more efficient technique to handle concurrent checkpoint initiations can be found in [27]. As multiple concurrent checkpoint initiation is orthogonal to our discussion, we only briefly mention the main features of [27]. When a process receives its first request for the checkpointing initiated by another process, it takes a local checkpoint and propagates the request. All local checkpoints taken by the participating processes for a checkpointing initiation collectively form a global checkpoint. The state information collected by each independent checkpointing is combined. The combination is driven by the fact that the union of consistent global checkpoints is also a consistent global checkpoint. The checkpoint thus generated is more recent than each of the checkpoints collected independently, and also more recent than that collected by [31]. Therefore, the amount of computation lost during rollback, after process failures, is minimized.

\subsection{Handling Failures during Checkpointing}

Since $M H$ s are more prone to failure, there is a possibility that, during the checkpointing activity, an $M H$ fails and all processes running on it also fail. We assume that if a process fails, some processes that try to communicate with it get to know of the failure. If the failed process is not the checkpointing initiator, the simplest way to deal with failures is to use abort messages similar to the approach in [19], [ 28]. More specifically, the process detecting the failures notifies the initiator, which broadcasts abort messages to all processes participating in the current checkpointing. These processes discard their checkpoints (tentative or mutable) and restore some variables such as sent, old_csn, $R$, etc, on receiving the abort messages. If the failed process is the coordinator and the failure occurred before the process sent out commit or abort messages, on restarting after failure, it broadcasts an abort corresponding to its checkpoint initiation. If the process had failed after broadcasting a commit or abort, it does not do anything more for that checkpoint initiation.

The above approach may not have good performance since the whole checkpointing aborts even when only one participating process fails. We would like to use a more efficient approach proposed by Kim and Park [18]. In their approach, processes can commit their tentative checkpoints when none of the processes on which they depend fails. Then, the consistent recovery line is advanced for those processes that committed their checkpoints. Certainly, the initiator and other processes which depend on the failed process have to abort their checkpointing and discard their tentative (mutable) checkpoints, as in [19]. In this way, the checkpoint-commit decision can be made locally so that the total abort of the checkpointing is avoided. In other words, when a process involved in a checkpointing coordination fails, the processes not affected by the failed one can make their decisions. Note that the protocols in [19] abort the whole checkpointing activity in case of failure.

In mobile computing systems, since wireless channels are more likely to suffer from intermittent errors, failure detection in wireless networks should be different from that in static networks. More information on how to deal with process failures can be found in [20], [24], [28]. Since failure detection and failure recovery are orthogonal to our discussion, we will not discuss it further.

\section{Correctness Proof}

In Section 3.2, $R_{i}$ represents all dependency relations in the current checkpointing period. Due to the introduction of mutable checkpoints, $R_{i}$ may represent the dependency relations after the last mutable checkpoint. To simplify the proof, in the following, $R_{i}$ means the first parameter of 


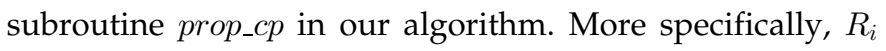
should be $C P_{i} . R$ if there is a mutable checkpoint.

Theorem 1. The algorithm creates a consistent global checkpoint.

Proof. We prove this by contradiction. Assume that the global state of the system is inconsistent at a time instance. Then, there must be a pair of processes $P_{i}$ and $P_{j}$ such that at least one message $m$ has been sent from $P_{j}$ after $P_{j}^{\prime}$ s last checkpoint and has been received by $P_{i}$ before $P_{i}$ 's last checkpoint. Since $R_{i}[j]=1$ when $P_{i}$ takes its checkpoint, $P_{i}$ sends a checkpoint request to $P_{j}$ or a process $P_{k}$ has sent the request to $P_{j}$ if $M R[j] . c s n \geq \operatorname{csn}_{i}[j]$. Thus, at least one checkpoint request has been sent to $P_{j}$. If $P_{j}$ runs on an MSS, the underlying network routes the request to it. If $P_{j}$ runs on an $M H_{i}$, which is in $M S S_{p}$ 's cell, there are three possibilities when the request reaches $M S S_{p}$ :

Case 1: If $M H_{i}$ is still connected to $M S S_{p}$, the request is forwarded to $M H_{i}$ and then to $P_{j}$.

Case 2: $M H_{i}$ has moved to $M S S_{q}$ (handoff). $M S S_{p}$ forwards the request to $M S S_{q}$, which forwards it to $M H_{i}$ and then to $P_{j}$ by the underlying routing protocol.

Case 3: $M H_{i}$ is disconnected from the network. $M S S_{p}$ takes a checkpoint on behalf of $P_{j}$ by converting disconnect_checkpoint $_{j}$ into $P_{j}^{\prime}$ 's new checkpoint (as explained in Section 2.2). Since $P_{j}$ cannot send any message after disconnection, it must have sent $m$ before its disconnection. Thus, the sending of $m$ is recorded in disconnect_checkpoint ${ }_{j}$. A contradiction.

In Case 1 and Case 2, when $P_{j}$ receives the request, if req_csn $<$ old_csn $_{j}$, no matter whether the request comes from $P_{i}$ or $P_{k}$ (if the request comes from $P_{k}$ :

$$
\begin{aligned}
& \left(\text { csn }_{i}[j] \leq M R[j] . c s n\right) \wedge\left(M R[j] . c s n=r e q \_c s n\right) \\
& \left.\wedge\left(r e q \_c s n<\text { old_csn }_{j}\right) \Longrightarrow c s n_{i}[j]<\text { old_csn }_{j}\right)
\end{aligned}
$$

$P_{j}$ has already taken a checkpoint after sending $m$. Thus, the sending of $m$ is recorded at $P_{j}$. If req_csn $\geq$ old_csn (the request may come from $P_{i}$ or $P_{k}$ ), there are two possibilities:

Case 1: own_trigger $\neq$ msg.trigger. There are two possibilities for $P_{j}$ to take a checkpoint:

1.1. The checkpoint is taken after the sending of $m$. Then:

- $\quad \operatorname{send}(m)$ at $P_{j} \longrightarrow^{1}$ receive $(\mathrm{m})$ at $P_{i}$

- $\quad$ receive $(\mathrm{m})$ at $P_{i} \longrightarrow$ checkpoint taken at $P_{i}$

- checkpoint taken at $P_{i} \longrightarrow$ request sent by $P_{i}$ to $P_{j}$

- $\quad$ request sent by $P_{i}$ to $P_{j} \longrightarrow$ checkpoint taken at $P_{j}$

Using the transitivity property of $\longrightarrow$, we have: send $(\mathrm{m})$ at $P_{j} \longrightarrow$ checkpoint taken at $P_{j}$. Thus, the sending of $m$ is recorded at $P_{j}$

1.2. The checkpoint is taken before the sending of $m$. As a result, $P_{j}$ increases $c s n_{j}[j]$ before it sends $m$ to $P_{i}$ and, hence, m.csn $>c s n_{i}[j]$. There are two possible situations:

1. $\longrightarrow$ is the "happened before" relation described in [22]
1.2.1.

$P_{j}$ has finished its checkpointing process (the last checkpoint) before it sends $m$. Hence, $P_{i}$ does not need to take a checkpoint when it receives $m$ and then the reception of $m$ is not recorded in the last checkpoint of $P_{i}$.

1.2.2.

$P_{j}$ has not finished its checkpointing process before it sends $m$. If $P_{i}$ does not need to take a mutable checkpoint before processing $m$, the reception of $m$ cannot be recorded in the last checkpoint of $P_{i}$. If $P_{i}$ takes a mutable checkpoint before processing $m$, when $P_{i}$ receives the request for this checkpoint initiation, $P_{i}$ turns the mutable checkpoint into a tentative checkpoint. Certainly, the reception of $m$ is still not recorded in the last checkpoint of $P_{i}$.

Case 2: own_trigger $=$ msg.trigger. In this case, $P_{j}$ has taken a mutable checkpoint or a tentative checkpoint. There are two possibilities:

2.1. The checkpoint is taken after the sending of $m$. If the checkpoint is a mutable checkpoint, on receipt of the request, it is changed to a tentative checkpoint. Thus, the sending of $m$ is recorded.

2.2. The checkpoint is taken before the sending of $m$. Similar to Case 1.2, we get contradictions.

Lemma 1. Every process inherits at most one checkpoint request to take a checkpoint.

Proof. After a process $P_{i}$ inherits a checkpoint request, it changes its own_trigger to the trigger attached with the request and takes a checkpoint (or make a mutable checkpoint permanent). Later, when it receives other checkpoint requests corresponding to this checkpoint initiation, own_trigger $=m s g_{-}$trigger and $P_{i}$ cannot take a mutable checkpoint, i.e., $C P_{i}$.trigger $\neq$ own_trigger. Thus, $P_{i}$ cannot take a checkpoint on receipt of other requests corresponding to the same checkpoint initiation, that is, it does not inherit any request other than the first one.

In order to prove that our nonblocking checkpointing algorithm terminates, we introduce the following notations:

- $\quad W($ request $)$ : the weight carried by a request message.

- $W($ reply $)$ : the weight carried by a reply message.

- $W\left(P_{\text {init }}\right)$ : the weight at the initiator.

- $W\left(P_{\text {other }}\right)$ : the weight at a process other than the initiator.

Lemma 2. During a checkpointing process, the following invariant holds:

$$
\begin{gathered}
W\left(P_{\text {init }}\right)+\sum_{\forall \text { request }} W(\text { request })+\sum_{\forall \text { reply }} W(\text { reply }) 2 \\
+\sum_{\forall P_{\text {other }}} W\left(P_{\text {other }}\right)=1 .
\end{gathered}
$$


Proof. When $P_{\text {init }}$ initiates a checkpointing process, $W\left(P_{\text {init }}\right)=1$, no weight is associated with other processes, and no request or reply messages are in transit. Hence, the invariant holds. During the checkpointing process, the initiator sends out a portion of its weight in each outgoing request message. Therefore,

$$
\sum_{\forall \text { request }} W(\text { request })+W\left(P_{\text {init }}\right)=1 .
$$

When a process $P_{i}$ receives a checkpoint request, there are two possibilities:

Case 1: If $P_{i}$ needs to take a tentative checkpoint or to turn a mutable checkpoint into a tentative checkpoint, a part of the received weight is propagated to other processes in the request messages and the rest of the weight is sent to the initiator in a reply.

Case 2: If $P_{i}$ does not need to take a tentative checkpoint or turn a mutable checkpoint into a tentative checkpoint, the entire received weight is sent back to the initiator in a reply.

Therefore, no portion of the weight in a request is retained by $P_{i}$. At any instant of time during the checkpointing process, request and reply messages may be in transit and some noninitiator processes may have nonzero weights. However, no extra weight is created or deleted at any noninitiator process. Thus, the invariant holds.

Theorem 2. The proposed checkpointing algorithm terminates within a finite time.

Proof. In our algorithm, a process only propagates request messages when it inherits a request. Based on Lemma 1, every process inherits at most one request to take a checkpoint and then each process propagates the received request at most once. Since the number of processes in the system is finite, the number of request messages generated are finite. As message propagation delay is bounded, within a finite time after the checkpoint initiation, no new request messages will be generated and all such messages generated in the past have been delivered by the receivers. After this point of time, say $T$, the following assertion is true:

$$
\sum_{\forall \text { request }} W(\text { request })=0 .
$$

On the receipt of a request, a noninitiator process immediately sends out the weight received in the request on the outgoing request messages or reply messages. Thus, within a finite time after $T$, the weight of all noninitiator processes becomes zero. As there are no more request messages in the system, noninitiator processes cannot acquire any weight in the future. After this point of time, say $T^{\prime}>T$, the following assertion is true:

$$
\sum_{\forall P_{\text {other }}} W\left(P_{\text {other }}\right)=0
$$

As message propagation delay is finite, all reply messages will be received by the initiator within a finite time after $T^{\prime}$. As there are no more request messages, no new reply will be generated. Hence, after time, say $T^{\prime \prime}>T^{\prime}$, the following assertion is true:

$$
\sum_{\forall r e p l y} W(\text { reply })=0 .
$$

Based on Lemma 2:

$$
\begin{aligned}
W\left(P_{\text {init }}\right) & +\sum_{\forall \text { request }} W(\text { request }) \\
& +\sum_{\forall \text { reply }} W(\text { reply })+\sum_{\forall P_{\text {other }}} W\left(P_{\text {other }}\right)=1 .
\end{aligned}
$$

After time $T^{\prime \prime}$, since $T^{\prime \prime}>T^{\prime}>T$, assertions (1), (2), and (3) are all true. Thus, $W\left(P_{\text {init }}\right)=1$. At this point, the initiator sends commit messages to the processes that took checkpoints. A noninitiator process receives the commit message within a finite time. Therefore, the checkpointing algorithm terminates within a finite time.

We now show that the number of processes that take new tentative (permanent) checkpoints during the execution of our algorithm is minimal. Based on Lemma 1, a process takes at most one checkpoint corresponding to a checkpointing process. Let $\mathcal{P}=\left\{P_{0}, P_{1}, \cdots, P_{k}\right\}$ be the set of processes that take new checkpoints during the execution of our algorithm, where $P_{0}$ is the initiator. Let $\mathcal{C}(\mathcal{P})=$ $\left\{C\left(P_{0}\right), C\left(P_{1}\right), \cdots, C\left(P_{k}\right)\right\}$ be the new checkpoints taken by processes in $\mathcal{P}$.

When a process receives a checkpoint request, it asks all processes on which it depends to take checkpoints. The process receiving the request should take a checkpoint as soon as possible since the longer it waits, the more processes will have a dependency relation with it and then more processes need to take checkpoints. If the initiator knows all processes on which it depends, it can send checkpoint requests to them at once and then save the time of tracing the dependency tree. Some techniques [6], [28] exist to approximate this approach. However, it increases run time overhead since extra information has to be appended with the computation messages. Since the message delay is far less than the time between two checkpoint intervals, we do not consider the extra checkpoints resulting from the checkpoint request delay. Our algorithm can also use the techniques in [6], [28] to reduce the number of extra checkpoints, but, as we discussed, it is not valuable due to increased run time overhead.

We define an alternate set of checkpoints: $\mathcal{C}^{\prime}(\mathcal{P})=\left\{C^{\prime}\left(P_{0}\right), C^{\prime}\left(P_{1}\right), \cdots, C^{\prime}\left(P_{k}\right)\right\}$, where $C^{\prime}\left(P_{0}\right)=C\left(P_{0}\right)$ and $C^{\prime}\left(P_{i}\right)(1 \leq i \leq k)$ is either $C\left(P_{i}\right)$ or the checkpoint $P_{i}$ had taken before executing our algorithm. If $C^{\prime}\left(P_{i}\right)$ is a new checkpoint, as we discussed, it should be taken as soon as possible and then it is equal to $C\left(P_{i}\right)$ without considering the checkpoint request delay.

Theroem 3. $\mathcal{C}^{\prime}(\mathcal{P})$ is consistent if and only if $\mathcal{C}^{\prime}(\mathcal{P})=\mathcal{C}(\mathcal{P})$.

Proof. The if part directly comes from Theorem 1. We now prove the only if part. The execution of our algorithm 
imposes a " $P_{i}$ inherits a request from $P_{j}$ " (defined in Section 3.2) relation on the set of processes. Since this relation is noncircular (based on Lemma 1) and there is only one initiator, it can be represented as a tree $T$ : The root of $T$ is the initiator and $P_{j}$ is a child of $P_{i}$ if and only if $P_{j}$ inherits a request from $P_{i}$. If $P_{j} \in T$, it must take a new checkpoint during the execution of the algorithm; hence, $P_{j} \in \mathcal{P}$. If $P_{j} \in \mathcal{P}$, either $P_{j}$ is the initiator or it inherits a request; hence, $P_{j} \in T$. Therefore, $P_{j} \in T$ if and only if $P_{j} \in \mathcal{P}$.

Our proof is by contradiction. Suppose $\mathcal{C}^{\prime}(\mathcal{P}) \neq$ $\mathcal{C}(\mathcal{P})$ and $\mathcal{C}^{\prime}(\mathcal{P})$ is consistent. Let $P_{j} \in \mathcal{P}$ such that $C^{\prime}\left(P_{j}\right) \neq C\left(P_{j}\right)$. Note that $P_{j} \neq P_{0}$ and there exists a path from $P_{0}$ to $P_{k}$ in $T$. Since $C^{\prime}\left(P_{0}\right)=C\left(P_{0}\right)$, there is an edge $\left(P_{i}, P_{j}\right)$ on this path such that $C^{\prime}\left(P_{i}\right)=$ $C\left(P_{i}\right) \wedge C^{\prime}\left(P_{j}\right) \neq C\left(P_{j}\right)$. Let $m$ be the last message $P_{i}$ receives from $P_{j}$. Since $P_{j}$ inherits $P_{i}$ 's request, we have $r e q_{-} c s n \geq$ old $_{-} c s n_{j}$ (req_csn is appended with the request) and the receipt of $m$ is recorded in $C\left(P_{i}\right)$ (or $C^{\prime}\left(P_{i}\right)$ ). Also, the sending of $m$ is recorded in $C\left(P_{j}\right)$. If $C\left(P_{j}\right) \neq C^{\prime}\left(P_{j}\right), C^{\prime}\left(P_{j}\right)$ is the checkpoint $P_{j}$ had before executing the algorithm and then the sending of $m$ is not recorded in $C^{\prime}\left(P_{j}\right)$. Thus, $\mathcal{C}^{\prime}(\mathcal{P})$ is not a consistent set of checkpoints. A contradiction.

\section{A Performance Evaluation}

A mutable checkpoint is redundant if it is not turned into a tentative checkpoint. In this section, to evaluate the performance of our algorithm, we first use simulations to measure the number of redundant mutable checkpoints taken during the checkpointing time, which is the duration of a checkpointing process, from the initiation to the termination. Then, we compare our algorithm with other algorithms in the literature.

\subsection{Simulation Model}

A system with $N M H$ s connected through a wireless LAN is simulated. Each $M H$ has one process running on it and $N$ is equal to 16. The wireless LAN has a bandwidth of $2 \mathrm{Mbps}$, which follows IEEE 802.11 standard [11]. The length of each computation message is $1 K B$; Thus, the transmission delay of each computation message is $8 * 1 / 2=4 \mathrm{~ms}$. The length of each system message is 50 Bytes. Thus, the transmission delay of each system message is $0.05 * 8 / 2=0.2 \mathrm{~ms}$. The size of a checkpoint is $1 M B$ [13]. We can use incremental checkpointing [13] to reduce the amount of data that must be written on the stable storage; that is, only the pages of the address space that have been modified since the previous checkpoint are transferred to the MSS. As a result, we assume that only $512 \mathrm{~KB}$ are transmitted over the wireless link in order to take a tentative checkpoint which needs $0.5 * 8 / 2=2 s$ (disk access time is not counted). In today's technology, Pentium $600 \mathrm{MHz}$ laptops with $128 \mathrm{MB}$ memory are becoming popular. Thus, we assume mutable checkpoints are saved in the main memory. Since processor speed is much faster, the main memory is the bottleneck. Suppose a 64bit wide memory bus with $100 \mathrm{MHz}$ bus speed is used. Thus, it needs about $\frac{1 * 2}{100 * 8}=2.5 \mathrm{~ms}$ to save a mutable checkpoint. (If memory block copy is supported, the time can be further reduced.) A checkpoint is scheduled at each process with an interval of 900 seconds. If a process takes a checkpoint before its scheduled checkpoint time, the next checkpoint will be scheduled $900 \mathrm{~s}$ after that time. For simplicity, concurrent initiation, handoff, and failures are not considered.

Each process sends out computation messages with the time interval following an exponential distribution. The message receiving pattern is considered in two computation environments: point-to-point communication and group communication. In the point-to-point communication, the destination of each message is uniformly distributed among all processes. In the group communication, processes are arranged into four groups and each group has a group leader. For intragroup communication, the destination of each message is a uniformly distributed random variable among all group members. Only group leaders can have intergroup communication, where the destination of each message is a uniformly distributed random variable among all group leaders.

\subsection{Simulation Results}

Since saving checkpoints takes a long time, in order not to block the process's execution, we use precopying [17], that is, the pages are copied to a separate area in the main memory and are then written from there to the stable storage. This is similar to saving a mutable checkpoint first and then turning it into a tentative checkpoint. Thus, we do not measure the number of mutable checkpoints that will be turned into tentative checkpoints. We measure the number of tentative checkpoints and the number of redundant mutable checkpoints for each checkpoint initiation under various message sending rates. The mean value of a measured parameter is obtained by collecting a large number of samples such that the confidence interval is reasonably small. In most cases, the 95 percent confidence interval for the measured data is less than 10 percenet of the sample mean.

\subsubsection{Point-to-Point Communication}

As shown in Fig. 5, the number of tentative checkpoints for each checkpoint initiation increases as the message sending rate increases. Since the message receiving event is uniformly distributed, a process is more likely to receive a message from other processes when the message sending rate increases. Thus, it is more likely to have a dependency relationship with the initiator and thus is more likely to take a tentative checkpoint.

In Fig. 5, when the message sending rate increases, the number of redundant mutable checkpoints for each checkpoint initiation increases at first and then decreases and it is always less than 4 percent of the number of tentative checkpoints. This can be explained as follows: A process takes a mutable checkpoint only when it receives a computation message before it receives the checkpoint request during the checkpointing time. It takes a tentative checkpoint if it has received messages that created dependency relationships with the initiator during the checkpoint interval. Since the checkpointing time (at most $2 * 16=32 s$ long) is much less than the checkpoint interval (900s), in general, a process takes much fewer redundant mutable checkpoints than tentative checkpoints. If the 


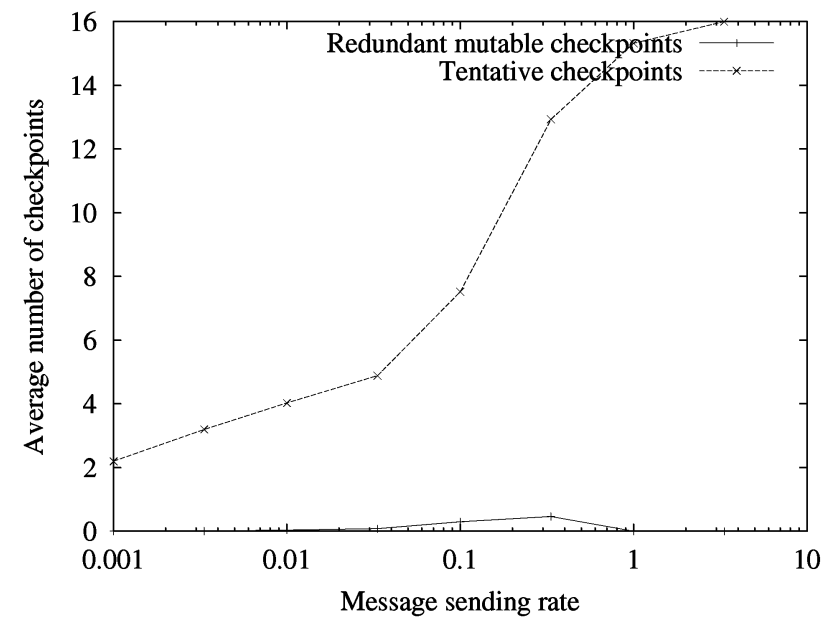

Fig. 5. The number of checkpoints in a point-to-point communication environment

message sending rate is low, processes have low probability of sending messages and they have low probability of receiving messages during the checkpointing time. Thus, they have low probability of taking mutable checkpoints. If the message sending rate is high, it is more likely for a process to receive a message and take a mutable checkpoint during the checkpointing time. The mutable checkpoint is also more likely to be turned into a tentative checkpoint and then it is not a redundant mutable checkpoint. According to our algorithm, the initiator quickly propagates the checkpoint request; thus, a process is less likely to receive a computation message before the checkpoint request during the checkpointing time and it is less likely to take a mutable checkpoint.

\subsubsection{Group Communication}

Fig. 6 shows the number of checkpoints in a group communication environment. Besides changing the intragroup message sending rate, a group leader also changes its intergroup message sending rate. On the left side of Fig. 6, for a group leader, the intragroup message sending rate is 1,000 times faster than the intergroup message sending rate; while on the right side of Fig. 6, the intragroup message sending rate is 10,000 times faster. As can be seen, with group communication, the number of tentative checkpoints and redundant mutable checkpoints on the right graph is less than that on the left graph and they are smaller than those in the point-to-point communication. In a group communication, when a process initiates a checkpointing process, processes in other groups have low probability of receiving messages from any process in the initiator's group. Thus, they are less likely to have dependency relationships with the initiator; that is, they have low probability of taking tentative checkpoints or redundant mutable checkpoints.

\subsection{Comparison with Other Algorithms}

The following notations are used to compare our algorithm with other algorithms.

\section{Notations:}

- $C_{a i r}$ : cost of sending a message from one process to another process.

- $C_{\text {broad: }}$ : cost of broadcasting a message to all processes.

- $T_{\text {disk }}$ : delay incurred in saving a checkpoint on the stable storage in an MSS.

- $T_{\text {data }}$ : delay incurred in transferring a checkpoint from an $M H$ to its MSS.

- $\quad T_{m s g}$ : delay incurred by system messages during a checkpointing process.

- $\quad T_{c h}$ : the checkpointing time. $T_{c h}=T_{m s g}+T_{\text {data }}+T_{\text {disk }}$.

- $\quad N_{\text {min }}, N, N_{\text {muta }}, N_{\text {dep }}: N_{\min }$ is the number of processes that need to take checkpoints using the Koo-Toueg algorithm [19]. $N$ is the total number of processes in the system. $N_{\text {muta }}$ is the number of redundant mutable checkpoints during a checkpointing process. $N_{d e p}$ is the average number of processes on which a process depends. Note that $1 \leq N_{d e p} \leq$ $N-1$.
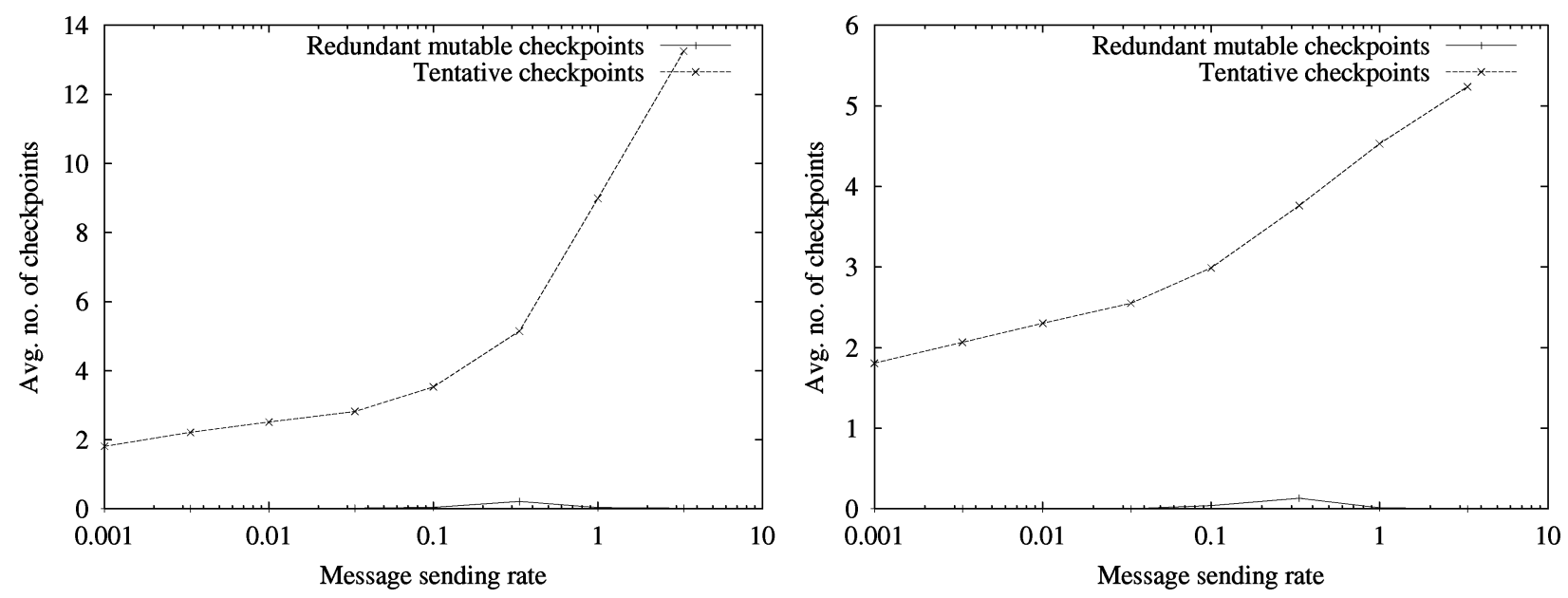

Fig. 6. The number of checkpoints in a group communication environment. On the left figure, for a group leader, the intragroup message sending rate is 1000 times faster than the intergroup message sending rate. On the right figure, the intragroup message sending rate is 10000 times faster than the intergroup message sending rate. 
TABLE 1

A Comparison of System Performance.

\begin{tabular}{|c|c|c|c|c|c|}
\hline Algorithm & Checkpoints & Blocking time & Output commit & Messages & Distributed \\
\hline 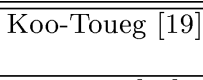 & $\overline{N_{\min }}$ & $\begin{array}{l}N_{\min } * T_{c h}=N_{\min } * \\
\left(T_{m s g}+T_{\text {data }}+T_{\text {disk }}\right) \\
\end{array}$ & 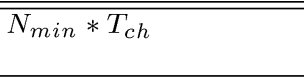 & 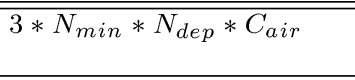 & $\overline{\text { Yes }}$ \\
\hline Elnozahy [13] & $N$ & 0 & $N * T_{c h}$ & $2 * C_{\text {broad }}+N * C_{a i r}$ & No \\
\hline Our algorithm & $N_{\min }$ & 0 & $\begin{array}{l}\left(N_{\text {min }}+N_{\text {muta }}\right) * T_{c h} \\
\approx N_{\text {min }} * T_{c h}\end{array}$ & $\begin{array}{l}\approx 2 * N_{\min } * C_{a i r}+ \\
\min \left(N_{\min } * C_{a i r}, C_{\text {broad }}\right)\end{array}$ & Yes \\
\hline
\end{tabular}

We use five parameters to evaluate the performance of a checkpointing algorithm: the number of tentative checkpoints required during a checkpointing process, the blocking time (in the worst case), the system message overhead, whether the algorithm is distributed or not, and the output commit delay, which is the delay incurred before the system commits to the outside world. The outside world consists of everything with which processes can communicate that does not participate in the system's rollback-recovery, such as the user's workstation display or even the file system if no special support is available for rolling back the contents of files. Messages sent to the outside world must be delayed until the system can guarantee that the message will never be "unsent" as a result of processes rolling back to recover from any possible future failure. If the sender is forced to roll back to a state before the message was sent, recovery to a consistent system state may be impossible since the outside world cannot, in general, be rolled back. Once the system can meet this guarantee, the message may be committed by releasing it to the outside world. Generally, if a process needs output commit, it initiates a checkpointing process. Thus, the output commit delay equals the duration of the checkpointing process.

\subsubsection{Performance of Our Algorithm}

It is easy to see that our algorithm is distributed and the blocking time is 0 .

The number of tentative checkpoints: Based on the result of Theorem 3, our algorithm forces only a minimum number of processes to save checkpoints on the stable storage.

The output commit delay: From the simulation results, the number of redundant mutable checkpoints is less than 4 percent of the number of tentative checkpoints. Based on the simulation parameters, the delay of taking a mutable checkpoint is almost 1,000 times shorter than that of taking a tentative checkpoint. Thus, the output commit delay of our algorithm is approximately $N_{\min } * T_{c h}$. Note that, for some applications, the checkpoint size is pretty small and the wireless network may have high bandwidth in the future, but, at that time, the memory bus bandwidth also becomes larger. Moreover, at that time, the disk access delay, which is difficult to reduce, may dominate the checkpointing time. Thus, the delay of taking a mutable checkpoint is still significantly shorter than that of taking a tentative checkpoint.

The system message overhead: In the first phase, a process taking a tentative checkpoint needs two system messages: request and reply. A process may receive more than one request for the same checkpoint initiation from different processes. However, we have used some techniques to reduce the occurence of this kind of situation. Thus, the system message overhead is approximately $2 * N_{\min } * C_{\text {air }}$ in the first phase. In the second phase, we hope to get the advantages of the update approach and the broadcast approach by system tuning. Thus, the system message overhead is approximately $\min \left(N_{\min } * C_{a i r}, C_{\text {broad }}\right)$ in the second phase.

\subsubsection{Comparison to Other Algorithms}

Table 1 compares our algorithm with two representative approaches for coordinated checkpointing. The Koo-Toueg algorithm [19] has the lowest overhead (based on our five parameters) among the blocking algorithms [3], [10], [12], [18], [19], [23], [29] which try to minimize the number of synchronization messages and the number of checkpoints. The algorithm in [13] has the lowest overhead (based on our five parameters) among the nonblocking algorithms [9], [13], [21], [30]. We do not compare our algorithm with the Prakash-Singhal algorithm since it may result in inconsistencies and there is no easy solution to fix it without increasing overhead.

As shown in Table 1, when compared to the Koo-Toueg algorithm, our algorithm reduces the message overhead from $3 * N_{\text {min }} * N_{d e p} * C_{a i r}\left(1 \leq N_{d e p} \leq N-1\right)$ to $2 * N_{\text {min }}$ $* C_{\text {air }}+\min \left(N_{\text {min }} * C_{\text {air }}, C_{\text {broad }}\right)$. When $N_{\text {min }}=N$, the message reduction can be from $O\left(N^{2}\right)$ to $O(N)$. Our algorithm reduces the blocking time from $N_{\min } * T_{c h}$ to 0 . In the worst case, $N_{\min }=N$. Consider our simulation parameters: $N=$ 16 and $T_{c h}=2 s$, the blocking time will be $32 s$, i.e., all processes cannot do anything for half a minute in the KooToueg algorithm, which significantly reduces the system performance. Compared to [13], our algorithm forces only a minimum number of processes to take their checkpoints on stable storage. Note that there may be many applications running in the system: Some of them have higher reliability requirement and others do not. In a heterogeneous environment, some $\mathrm{MHs}$ may be more prone to failures than others. Moreover, different processes may run at their own speed and they may only communicate with a group of processes. As a result, some processes may need to take checkpoints more frequently than others. However, the algorithm in [13] forces all processes in the system to take checkpoints for each checkpoint initiation. Thus, our algorithm significantly reduces the message overhead and checkpointing overhead compared to [13]. Furthermore, in the case of output commit, our algorithm has much shorter delay compared to [13] since our algorithm requires fewer processes to take checkpoints before committing to the outside world. It seems that our algorithm needs more system messages than [13]. However, the algorithm in [13] is a centralized algorithm and there is no easy way to make 
it distributed without significantly increasing message overhead. Since some processes may be in the doze mode, broadcast may waste their energy and processor power. More importantly, the system message is relatively small and the overhead of system messages is much smaller compared to the overhead of saving checkpoints on the stable storage.

\section{Related Work}

The first coordinated checkpointing algorithm was presented in [3]. However, it assumes that all communications between processes are atomic, which is too restrictive. The Koo-Toueg algorithm [19] relaxes this assumption. In this algorithm, only those processes that have communicated with the checkpoint initiator either directly or indirectly since the last checkpoint need to take new checkpoints. Thus, it reduces the number of synchronization messages and the number of checkpoints. Later, Leu and Bhargava [23] presented an algorithm which is resilient to multiple process failures and does not assume that the channel is FIFO, which is necessary in [19]. However, these two algorithms [19], [23] assume a complex scheme (such as slide window) to deal with the message loss problem and do not consider lost messages in checkpointing and recovery. Deng and Park [12] proposed an algorithm to address both orphan messages and lost messages.

In Koo and Toueg's algorithm [19], if any of the involved processes is not able to or not willing to take a checkpoint, the entire checkpointing process is aborted. Kim and Park [18] proposed an improved scheme that allows the new checkpoints in some subtrees to be committed, while the others are aborted.

To further reduce the system messages needed to synchronize the checkpointing, loosely synchronous clocks [10], [29] are used. More specifically, loosely synchronized checkpoint clocks can trigger the local checkpointing actions of all participating processes at approximately the same time without the need of broadcasting the checkpoint request by the initiator. However, a process taking a checkpoint needs to wait for a period that equals the sum of the maximum deviation between clocks and the maximum time to detect a failure in another process in the system.

All the above coordinated checkpointing algorithms [3], [10], [12], [18], [19], [23], [29] require processes to be blocked during checkpointing. Checkpointing includes the time to trace the dependency tree and to save the state of processes on the stable storage, which may be long. Therefore, blocking algorithms may dramatically reduce the performance of the system [5], [13].

The Chandy-Lamport algorithm [9] is the earliest nonblocking algorithm for coordinated checkpointing. However, in their algorithm, system messages (markers) are sent along all channels in the network during checkpointing. This leads to a message complexity of $O\left(N^{2}\right)$. Moreover, it requires all processes to take checkpoints and the channel must be FIFO. To relax the FIFO assumption, Lai and Yang [21] proposed another algorithm. In their algorithm, when a process takes a checkpoint, it piggybacks a checkpoint request (a flag) to the messages it sends out from each channel. The receiver checks the piggybacked message flag to see if there is a need to take a checkpoint before processing the message. If so, it takes a checkpoint before processing the message to avoid an inconsistency. To record the channel information, each process needs to maintain the entire message history on each channel as part of the local checkpoint. Thus, the space requirements of the algorithm may be large. Moreover, it requires all processes to take checkpoints, even though many of them are unnecessary.

The Elnozahy-Johnson-Zwaenepoel algorithm [13] uses the checkpoint sequence number to identify orphan messages, thus avoiding the need for processes to be blocked during checkpointing. However, this approach requires the initiator to communicate with all processes in the computation. The algorithm proposed by Silva and Silva [30] uses a similar idea as [13] except that the processes which did not communicate with others during the previous checkpoint interval do not need to take new checkpoints. Both algorithms [13], [30] assume that a distinguished initiator decides when to take a checkpoint. Therefore, they suffer from the disadvantages of centralized algorithms, such as one-site failure, traffic bottle-neck, etc. Moreover, their algorithms require almost all processes to take checkpoints, even though many of them are unnecessary. If they are modified to permit more processes to initiate checkpointing, which makes them distributed, the new algorithm suffers from another problem; in order to keep the checkpoint sequence number updated, any time a process takes a checkpoint, it has to notify all processes in the system. If each process can initiate a checkpointing process, the network would be flooded with control messages and processes might waste their time taking unnecessary checkpoints.

All the above algorithms follow two approaches to reduce the overhead associated with coordinated checkpointing algorithms: One is to minimize the number of synchronization messages and the number of checkpoints [3], [10], [12], [18], [19], [23], [29]; the other is to make checkpointing nonblocking [9], [13], [21], [30]. These two approaches were orthogonal in previous years until the Prakash-Singhal algorithm [28] combined them. However, their algorithm may result in an inconsistency in some situations [7], [8].

Acharya and Badrinath [1] were the first to present a checkpointing algorithm for mobile computing systems. In their uncoordinated checkpointing algorithm, an $\mathrm{MH}$ takes a local checkpoint whenever a message reception is preceded by a message sent at that $M H$. If the send and receive of messages are interleaved, the number of local checkpoints will be equal to half of the number of computation messages, which may degrade the system performance.

For other uncoordinated checkpointing algorithms, as described in [4], [32], every process may accumulate multiple local checkpoints and logs on the stable storage during normal operation. A checkpoint can be discarded if it is determined that it will no longer be needed for recovery. For this purpose, processes have to periodically 
broadcast the status of their logs on the stable storage. The number of local checkpoints depends on the frequency with which such checkpoints are taken and is an algorithm tuning parameter. An uncoordinated checkpointing approach is not suitable for mobile computing for a number of reasons. If the frequency of local checkpointing is high, each process will have multiple checkpoints, which requires a large amount of stable storage and introduces a lot of communication overhead in mobile computing systems. The stable storage and communication overheads can be reduced by taking local checkpoints less frequently. However, this will increase the recovery time as greater rollback and reply will be needed. Even though some algorithms [24], [35] were proposed to reduce the number of checkpoints to be saved on the stable storage, to ensure correctness, a process still needs to keep many more checkpoints in uncoordinated checkpointing algorithms than those in coordinated checkpointing algorithms. In the coordinated checkpointing algorithm presented in this paper, most of the time, each process needs to store only one permanent checkpoint on the stable storage and at most two checkpoints: a permanent and a tentative (or mutable) checkpoint only for the duration of the checkpointing. Generally speaking, uncoordinated checkpointing approaches suffer from the complexities of finding a consistent recovery line after the failure, the susceptibility to the domino effect, the high stable storage overhead of saving multiple checkpoints of each process, and the overhead of garbage collection. Thus, our coordinated checkpointing algorithm has many advantages over uncoordinated checkpointing algorithms.

\section{Conclusions}

Mobile computing is a rapidly emerging trend in distributed computing. A mobile computing system consists of mobile hosts (MHs) and mobile support stations (MSSs), connected by a communication network. The mobility of $\mathrm{MHs}$ in mobile computing systems generates many new constraints, such as handoffs, lack of stable storage, low communication bandwidth of a wireless channel, and energy conservation, which make the traditional checkpointing algorithm unsuitable. These new constraints require that the checkpointing algorithm should be nonblocking and only forces a minimum number of processes to take checkpoints. However, according to our previous result [7], [8], there does not exist a nonblocking algorithm which forces only a minimum number of processes to take their checkpoints. In order to design an efficient checkpointing algorithm for mobile computing systems, we introduced a new concept called "mutable checkpoint," which is neither a tentative checkpoint nor a permanent checkpoint, but it can be turned into a tentative checkpoint. Mutable checkpoints can be saved anywhere, e.g., the main memory or local disk of MHs. In this way, taking a mutable checkpoint avoids the overhead of transferring a large amount of data to the stable storage at MSSs over the wireless network. We also presented techniques to minimize the number of mutable checkpoints. Simulation results show that the overhead of taking mutable checkpoints is negligible. Based on mutable checkpoints, our nonblocking algorithm avoids the avalanche effect and forces only a minimum number of processes to take their checkpoints on the stable storage.

\section{REFERENCES}

[1] A. Acharya and B.R. Badrinath, "Checkpointing Distributed Applications on Mobil Computers," Proc. Third Int'l Conf. Parallel and Distributed Information Systems, Sept. 1994.

[2] I. Akyildiz, J. Mcnair, J. Ho, H. Uzunalioglu, and W. Wang, "Mobility Management in Next-Generation Wireless Systems," IEEE, vol. 87, no. 8. pp. 1347-1384, Aug. 1999.

[3] G. Barigazzi and L. Strigini, "Application-Transparent Setting of Recovery Points," Digest of Papers Fault-Tolerant Computing Systems-13, pp. 48-55, 1983.

[4] B. Bhargava and S. Lian, "Independent Checkpointing and Concurrent Rollback for Recovery in Distributed Systems," Proc. Seventh IEEE Symposium Reliable Distributed System, pp. 3-12, Oct. 1988.

[5] B. Bhargava, S.R. Lian, and P.J. Leu, "Experimental Evaluation of Concurrent Checkpointing and Rollback-Recovery Algorithms," Proc. Int'l Conf. Data Eng., pp. 182-189, 1990.

[6] G. Cao and M. Singhal, "Low-Cost Checkpointing with Mutable Checkpoints in Mobile Computing Systems," Proc. 18th Int'l Conf. Distributed Computing Systems, pp. 464-471, May 1998.

[7] G. Cao and M. Singhal, "On Coordinated Checkpointing in Distributed Systems," IEEE Trans. Parallel and Distributed System pp. 1213-1225, Dec. 1998.

[8] G. Cao and M. Singhal, "On the Impossibility of Min-Process NonBlocking Checkpointing and an Efficient Checkpointing Algorithm for Mobile Computing Systems," Proc. 27th Int'l Conf. on Parallel Processing, pp. 37-44, Aug. 1998.

[9] K.M. Chandy and L. Lamport, "Distributed Snapshots: Determining Global States of Distributed Systems," ACM Trans. Computer Systems, Feb. 1985.

[10] F. Cristian and F. Jahanian, "A Timestamp-Based Checkpointing Protocol for Long-Lived Distributed Computations," Proc. IEEE Symp. Reliable Distributed Systems, pp. 12-20, 1991.

[11] B. Crow, I. Widjaja, J. Kim, and P. Sakai, "IEEE 802. 11 Wireless Local Area Networks," IEEE Comm. Magazine, pp. 116-126, Sept. 1997.

[12] Y. Deng and E.K. Park, "Checkpointing and Rollback-Recovery Algorithms in Distributed Systems," J. Systems and Software, pp. 5971, Apr. 1994.

[13] E.N. Elnozahy, D.B. Johnson, and W. Zwaenepoel, "The Performance of Consistent Checkpointing," Proc. 11th Symp. Reliable Distributed Systems, pp. 86-95, Oct. 1992.

[14] G.H. Forman and J. Zahorjan, "The Challenges of Mobile Computing," Computer, pp. 38-47, Apr. 1994.

[15] J. Gray, Notes on Data Base Operating Systems, pp. 393-481, Springer-Verlag, 1979.

[16] S.T. Huang, "Detecting Termination of Distributed Computations by External Agents," Proc. Ninth Int'l Conf. Distributed Computing Systems, pp. 79-84, 1989.

[17] D. Johnson, "Distributed System Fault Tolerance Using Message Logging and Checkpointing," PhD Thesis, Rice Univ., Dec. 1989.

[18] J.L. Kim and T. Park, "An Efficient Protocol for Checkpointing Recovery in Distributed Systems," IEEE Trans. Parallel and Distributed Systems, pp. 955-960, Aug. 1993.

[19] R. Koo and S. Toueg, "Checkpointing and Rollback-Recovery for Distributed Systems," IEEE Trans. Software Eng., pp. 23-31, Jan. 1987.

[20] P. Krishna, N.H. Vaidya, and D.K. Pradhan, "Recovery in Distributed Mobile Environments," Proc. IEEE Workshop Advances in Parallel and Distributed System, Oct. 1993.

[21] T.H. Lai and T.H. Yang, "On Distributed Snapshots," Information Processing Letters, pp. 153-158, May 1987.

[22] L. Lamport, "Time, Clocks and Ordering of Events in Distributed Systems," Comm. of the ACM, July 1978. 
[23] P.Y. Leu and B. Bhargava, "Concurrent Robust Checkpointing and Recovery in Distributed Systems," Proc. Fourth IEEE Int'l. Conf. Data Eng., pp. 154-163, 1988.

[24] D. Manivannan and M. Singhal, "A Low-Overhead Recovery Technique Using Quasi-Synchronous Checkpointing," Proc. 16th Int'l Conf. Distributed Computing Systems, pp. 100-107, May 1996.

[25] R. Netzer and J. Xu, "Necessary and Sufficient Conditions for Consistent Global Snapshots," IEEE Trans. Parallel and Distributed Systems, pp. 165-169, Feb. 1995.

[26] C. Perkins, "Mobile IP," IEEE Comm. Magazine, vol. 35, pp. 84-99, May 1997.

[27] R. Prakash and M. Singhal, "Maximal Global Snapshot with Concurrent Initiators," Proc. Sixth IEEE Symp. Parallel and Distributed Processing, pp. 344-351, Oct. 1994.

[28] R. Prakash and M. Singhal, "Low-Cost Checkpointing and Failure Recovery in Mobile Computing Systems," IEEE Trans. Parallel and Distributed Systems, pp. 1035-1048, Oct. 1996.

[29] P. Ramanathan and K.G. Shin, "Use of Common Time Base for Checkpointing and Rollback Recovery in a Distributed System," IEEE Trans. Software Eng., pp. 571-583, June 1993.

[30] L.M. Silva and J.G. Silva, "Global Checkpointing for Distributed Programs," Proc. 11th Symp. Reliable Distributed Systems, pp. 155162, Oct. 1992

[31] M. Spezialetti and P. Kearns, "Efficient Distributed Snapshots," Proc. Sixth Int'l Conf. Distributed Computing Systems, pp. 382-388, 1986.

[32] R.E. Strom and S.A. Yemini, "Optimistic Recovery In Distributed Systems," ACM Trans. Computer Systems, pp. 204-226, Aug. 1985.

[33] F. Teraoka, Y. Yokote, and M. Tokoro, "A Network Architecture Providing Host Migration Transparency," Proc. ACM SIGCOMM '91, Sept. 1991.

[34] N. Vaidya, "Staggered Consistent Checkpointing," IEEE Trans. Parallel and Distributed Systems, vol. 10, no. 7, pp.694-702, July 1999.

[35] Y. Wang and W.K. Fuchs, "Lazy Checkpoint Coordination for Bounding Rollback Propagation," Proc. 12th Symp. Reliable Distributed Systems, pp. 78-85, Oct. 1993.

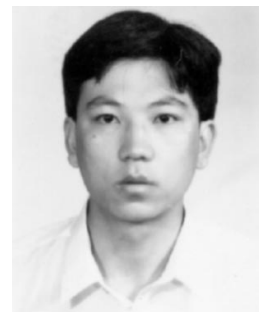

Guohong Cao received the BS degree from Xian Jiaotong University, Xian, China. He received the MS and $\mathrm{PhD}$ degrees in computer science from Ohio State University in 1997 and 1999, respectively. He was a recipient of the Presidential Fellowship at The Ohio State University. Since Fall 1999, he has been an assistant professor of computer science and engineering at Pennsylvania State University. His research interests include distributed faulttolerant computing, mobile computing, and wireless networks. He is a member of the IEEE.

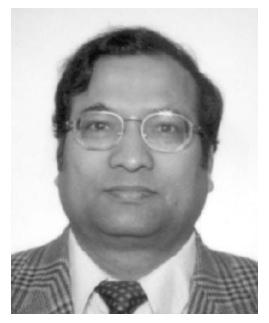

Mukesh Singhal received a B. Eng. degree in electronics and communication engineering with high distinction from the University of Roorkee, Roorkee, India, in 1980 and a PhD degree in computer science from the University of Maryland, College Park, in May 1986. He is a full professor of computer and information science at Ohio State University, Columbus. His current research interests include operating systems, database systems, distributed systems, mobile computing, high-speed networks, computer security, and performance modeling. He has published more than 100 refereed articles in these areas. He has coauthored two books titled Advanced Concepts in Operating Systems (McGraw-Hill, 1994) and Readings in Distributed Computing Systems (IEEE Press, 1993) He is currently the program director of the Operating Systems and Compilers Program at the National Science Foundation, US. He is a Fellow of the IEEE. 